\title{
Contre-jour
}

Cahiers littéraires

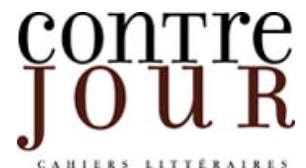

\section{Il aura (encore) parlé en nous avant nous}

\section{René Major}

Numéro 9, printemps 2006

À la mémoire de Jacques Derrida

URI : https://id.erudit.org/iderudit/631ac

Aller au sommaire du numéro

Éditeur(s)

Cahiers littéraires Contre-jour

ISSN

1705-0502 (imprimé)

1920-8812 (numérique)

Découvrir la revue

Citer cet article

Major, R. (2006). Il aura (encore) parlé en nous avant nous. Contre-jour, (9), 99-106. d'utilisation que vous pouvez consulter en ligne.

https://apropos.erudit.org/fr/usagers/politique-dutilisation/ 


\section{Il aura (encore) parlé en nous avant nous}

\section{René Major}

Depuis un an et demi, depuis que le verdict était tombé et qu'il l'avait confié à quelques amis...

(Le verdict, le mot verdict débute et termine un texte écrit il y a sept ans, «Un ver à soie ${ }^{1} »$ :

Avant le verdict, le mien, avant que tombant sur moi, il ne m'attire avec lui dans la chute, avant qu'il ne soit trop tard, ne point écrire $[\ldots]$

Et à la fin :

Un laps de temps, ce ne fut qu'un intervalle, presque rien, la diminution infinie d'un intervalle musical, et quelle note, quelle nouvelle, quelle musique. Le verdict. Comme si tout à coup le mal, rien de mal n'arrivait plus. Comme si rien de mal n'arrivait plus que la mort - ou seulement plus tard, trop tard, tellement plus tard.)

...oui, depuis que le verdict était tombé, le verdict qu'il avait, lui, maintes fois anticipé, chaque jour la pensée m'occupait qu'il faudrait croire à ce que je ne pouvais croire, qu'il faudrait penser ce qui était et restera impensable. Incapable de le penser, « c'est sur mon sort que je pleure, moi qui serai privé de la familiarité d'un pareil homme ». 
Lorsque Jacques Derrida écrit pour la première fois à la mort d'un ami, il précise qu'il fait ce qu'il s'était promis de ne jamais faire :

Mais ce que je croyais impossible, indécent, injustifiable, ce que dès longtemps, plus ou moins secrètement et résolument, je m'étais promis de ne jamais faire (souci de rigueur, de fidélité, si l'on veut, et parce que cette fois c'est trop grave), c'est d'écrire à la mort, non pas après, longtemps après la mort, en revenant, mais à la mort, à l'occasion de la mort [...].

Nous en sommes aujourd'hui à « cette fois c'est trop grave ", devant l'impossible, l'indécent, l'injustifiable. Et pourtant, il ajoute, dans un dialogue avec lui-même :

- Mais le silence alors? N'est-ce pas une autre blessure, une autre injure?

$-\grave{A}$ qui?

Oui, à qui faisons-nous présent et de quoi? Que faisons-nous quand nous échangeons ces discours? À quoi veillons-nous?

(Chaque fois..., 77)

À quoi veillons-nous et comment veiller ? Veiller à le porter et à être porté par lui. Ich muss dich tragen, dit le poème de Paul Celan que poursuit le dialogue ininterrompu. Veiller en parole ou en silence. Parler ou se taire : l'un et l'autre tout aussi impossibles. Partager notre tristesse pour être moins seuls. Ou plus seuls encore. Plus seuls ensemble.

Et pourtant, devant cette double impossibilité, il aura encore parlé en nous avant nous, en laissant un dernier mot, lu par son fils Pierre le mardi 12 octobre à Ris Orangis, nous enjoignant de sourire, de garder la mémoire des moments heureux.

Je sais la singularité qui aura marqué chacune de ses amitiés, dans un rapport chaque fois unique. Hélène [Cixous] et Marie-Louise [Mallet], ici à mes côtés, en témoignent. Comment en parler, sinon en évoquant quelques moments, comme il nous arrivait de le faire, avec un sourire complice ou malicieux, certains moments qui ont marqué notre longue amitié dont le secret essentiel me demeure inconnu et restera sans doute 
inconnaissable. Comme restent énigmatiques son attention au-delà de toute prévenance, sa générosité au-delà de tout ce que nous connaissons sous ce nom, aussi bien dans l'amitié que dans l'écriture, la bénédiction que ce fut de le connaître, la chance et la bénédiction d'une vie, la rencontre du dire juste et bien, du veridictum et du benedictum.

Je cède donc à l'indécence de dire ce que nous faisions ensemble. Il en parla lui-même un jour comme de la rencontre de deux dissidences.

Nous nous sommes trouvés un soir chez Anne et Emmanuel Clancier avec Maria Torok et Nicolas Abraham. C'était au début des années soixante. Nous avions trente ans. Que trente ans, dirais-je aujourd'hui. Peu de temps plus tard, j'assistais à la célèbre conférence que Jacques prononça à l'Institut de psychanalyse : “Freud et la scène de l'écriture », depuis laquelle il devenait explicite que la pensée de la déconstruction n'allait pas sans la prise en compte de ce que la psychanalyse appelle à penser, qui ne s'épuise ni dans la métaphysique ni dans la science, d'une écriture inhérente à la parole et, inversement, que la psychanalyse aurait à prendre la mesure de la déconstruction du logocentrisme, d'une radicalisation de la pensée de la trace, de la nécessité de soustraire les concepts de trace et de différance à toutes les oppositions conceptuelles classiques.

De l'apport questionnant de Derrida à la psychanalyse, qui ne cessera de jalonner toute son œuvre, nous sommes très loin encore d'avoir pris la mesure. À peine apercevons-nous une ébauche de cette mesure.

L'année où paraissait L'écriture et la différence (1967), je faisais ma première conférence à la Société psychanalytique de Paris. C'était sur le thème “ L'économie de la représentation ». Quelle ne fut pas ma surprise d'apercevoir Jacques dans l'assistance pendant que je parlais ! D'autant qu'il n'était pas évident de s'introduire dans ce milieu fermé. C'était un clin d'œil, et les quelques mots échangés après la conférence, un sourire qui n'allait cesser de nous accompagner.

Dès les années soixante-dix, je prenais mes distances avec ce milieu fermé où il était visible et pré-visible que les questions soulevées, que ce soit par "Freud et la scène de l'écriture ", par "Spéculer-sur "Freud" ", 
par “Le facteur de la vérité » (cette minutieuse lecture du Séminaire de Lacan sur La lettre volée de Poe), allaient rester sans lendemain. Il fallait donc inventer un lieu, un lieu sans résidence fixe, un espacement échappant à toute détermination existante et à toute réappropriation possible, un lieu rebelle à sa propre institutionnalisation, une khôra. Ce fut Confrontation. Un espace, une revue auxquels Jacques ne ménagea pas ses contributions. Je me souviens que lorsqu'il vint en ce lieu pour la première fois, il me mit en garde : "Ils vous feront payer cher notre amitié », me dit-il. Son intervention, sous la forme improvisée et dialoguée, portait sur l'effet "Confrontation ", son effet déstabilisateur, pour ne pas dire " déconstructeur ", par rapport aux quatre groupements d'analystes alors existants, leurs discours, leurs codes, leurs bords, les limites incertaines entre eux comme entre l'analyste et le non-analyste. Est-ce que cela peut exister, en toute rigueur, du non-analyste? À témoin cette rumeur, qui fait encore sourire, traversant une université américaine, selon laquelle Loewenstein, l'analyste de Lacan émigré aux États-Unis, était en analyse chez Derrida depuis plus de dix ans.

Vous vous étiez bien amusé, cher Jacques, et moi aussi, de cette tranche d'analyse de Loewenstein, pourtant déjà disparu, et de celle que pourrait encore faire Freud lui-même. Je relis cette séance du 21 novembre 1977, que vous avez reprise sous le titre « Du tout » dans La carte postale, de Socrate à Freud et au-delà. C'est une leçon d'humour de bout en bout. Non moins sérieuse pour autant si on pense aux jalons que vous posez pour un décryptage à venir du mouvement psychanalytique en France et ses conséquences théoriques, pratiques, institutionnelles. C'est ce qu'un week-end de travail autour de La carte postale devait inaugurer et qui fut publié sous le titre Affranchissement du transfert et de la lettre aux Éditions Confrontation. Ce qui m'intéressait tout particulièrement était de voir comment l'exclusion du narrateur dans la narration, au cours de l'analyse faite par Lacan, allait se retrouver à propos de la place de l'interprète dans l'interprétation quand l'enseignement lacanien à la même époque se portait sur " la direction de la cure » et quelles places viendront occuper dans cette histoire Freud, Marie Bonaparte, Loewenstein, Nacht et Lacan, au regard de celles qu'occupent le Roi, la Reine, le Préfet, le Ministre et Dupin dans le conte. 
Vous n'aurez pas boudé votre plaisir non plus, et je n'ai pas boudé le mien, lorsqu'en 1981 vous avez fait la conférence d'introduction, "Géopsychanalyse "and the rest of the world" ", à un Colloque francolatino-américain, en analysant les vagues et timides prises de position de l'Association internationale de psychanalyse au sujet de la torture et des droits de l'homme, alors que l'Argentine était en pleine dictature militaire et qu'une véritable réflexion de la psychanalyse sur le politique eût exigé tout autre chose. Je ne sais si nos vues communes sur le politique contribuèrent à un plus grand rapprochement. Nos relations, de fait, s'intensifièrent. Nous dînions souvent ensemble, à Ris ou à la maison, parfois au restaurant, le plus souvent à quatre, avec Marguerite et Chantal. Vous étiez alors entouré d'analystes mais, à la vérité, on ne sut jamais qui de nous quatre l'était ni quand il l'était.

En 1983, à la création du Collège, vous m'avez demandé d'assurer une direction de programme. Vous teniez à ce que la psychanalyse soit présente au Collège. Ce qui n'était pas l'avis d'autres membres fondateurs. Vous avez tenu bon et je vous en sais gré. Lorsque je fis une conférence à la Sorbonne, sous les auspices du Collège, " La raison depuis l'inconscient ”, vous m'avez introduit longuement sous le titre significatif « Ne pas oublier - la psychanalyse ", introduction et conférence qui furent publiées en anglais ${ }^{2}$ grâce à la vigilante attention de notre ami Geoffrey Bennington. Vous défendiez la psychanalyse contre ce qui était déjà « à l'ordre moral de l'ordre du jour ", une restauration honteuse et bâclée de la conscience du moi et de son autorité. Plus encore aujourd'hui qu'en 1988, cette plate restauration est quotidiennement revendiquée. L'an dernier à Vienne, j'ai découvert dans la maison de Freud un livre portant le même titre en allemand, Vergessen Wir nicht - die Psychoanalyse qui regroupe quelquesuns de vos textes des années quatre-vingt-dix sur la psychanalyse: "Résistances ", "Être juste avec Freud ", "Pour l'amour de Lacan ", suivis d'une étude de Hans-Dieter Gondek sur « Jacques Derrida et la psychanalyse " qui a pour titre «La séance continue . Ce titre reprend un mot de Freud à la mort de sa fille bien-aimée, Sophie, mot auquel vous avez consacré un long commentaire dans "Spéculer - sur "Freud" ". J'étais heureux de vous dire à mon retour de Vienne que Freud m'avait exprimé sa satisfaction de voir ce livre chez lui au 19 Bergasse. 
En relisant « Pour l'amour de Lacan », prononcé au colloque « Lacan avec les philosophes ", dont on sait que la tenue et la publication des Actes connurent une crise majeure qui témoignait elle-même de l'importance de l'événement, je mesure encore, s'il en était besoin, à la lecture du Postscriptum de Jacques, la rigueur de son argumentation dans l'indéfectible solidarité qu'il manifesta sans ambages sur les principes éthico-politiques que nous soutenions lorsqu'il était question de faire changer le titre d'un exposé (le mien) pour y effacer le nom propre qu'il contenait (le sien). Ceci pour redire que la place de Derrida dans l'histoire de la psychanalyse n'aura jamais été simple et toujours aussi difficilement acceptée par la philosophie que par la psychanalyse.

Des contributions de Jacques à la Société d'Histoire de la Psychiatrie et de la Psychanalyse, qu'il me soit permis d'évoquer celle, magistrale, qu'il offrit sur L'Archive en 1994 à Londres, sous les auspices du Freud Museum et du Courtauld Institute of Art où il montrait, dans une discussion très aiguë du Moïe de Yerushalmi, présent au colloque, comment on peut archiver dans le refoulement, refouler l'archive en archivant le refoulement et comment « nul mieux que Freud n'a éclairé ce que nous avons appelé le principe archontique de l'archive [...] [et] analysé, c'est-à-dire aussi déconstruit l'autorité du principe archontique ». Derrida partageait avec Freud l'impulsion intime du déchiffrement du désir intérieur de déchiffrer. Il reste que du secret lui-même, du secret d'une amitié, il ne peut y avoir d'archive : « Le secret, c'est la cendre même de l'archive, le lieu où il n'y a même plus de sens à dire "la cendre même" ou “à même la cendre”. » (Mal d'Archive, 148-149, 154)

Je passe sous silence de nombreux voyages que nous avons faits ensemble à Bordeaux, Lille, Toulouse, Madrid, Barcelone, les colloques de Cerisy autour de son travail que Marie-Louise Mallet, en remarquable musicienne, savait si bien orchestrer. C'était chaque fois un bonheur. Une chose moins connue, c'est la revue Contretemps que nous concevions ensemble, et avec Chantal, même s'il avait tenu à ce que sa place reste discrète. Le premier numéro comporte un article de lui, «Sauver les phénomènes ", qui n'a pas été repris dans ses livres. Le numéro $2 / 3$ fut l'occasion d'un événement littéraire unique. Il écrivit “ Un ver à soie » au 
cours d'un voyage en Amérique du sud en ayant apporté avec lui le texte d'Hélène Cixous, "Savoir ». Et c'est en lisant le texte d'Hélène relatant à la troisième personne une chirurgie de l'œil qu'il découvrait, lui qui la connaissait si bien depuis trente-trois ans, qu'elle était myope et qu'il n'avait pas vu jusque-là qu'elle ne le voyait pas la regarder.

En l'an 2000, Jacques s'adressait aux États Généraux de la psychanalyse, qui se tenaient à la Sorbonne, pour parler de la cruauté, pour dire aux psychanalystes que « Le seul discours qui puisse aujourd'hui revendiquer la chose de la cruauté psychique comme son affaire propre, ce serait bien ce qui s'appelle, depuis un siècle à peu près, la psychanalyse [...]. "Psychanalyse" serait le nom de ce qui, sans alibi théologique ou autre, se tournerait vers ce que la cruauté psychique aurait de plus propre. La psychanalyse [...] ce serait l'autre nom du "sans alibi". L'aveu d'un "sans alibi". Si c'était possible. "Son discours ouvrait, plus que jamais, “à la venue inconditionnelle de l'autre, à son événement inanticipable et sans horizon, sa mort ou la mort même [...] Peut-être au-delà de toute cruauté ». Il ajoutait : “ Et s'il y avait, parfois, de la cruauté à ne pas donner la mort? Et de l'amour à vouloir se donner la mort à deux, l'un à l'autre, l'un pour l'autre, simultanément ou non ? Et s'il y avait du "ça souffre cruellement en moi, en un moi" sans qu'on puisse jamais soupçonner quiconque d'exercer une cruauté ? de la vouloir ? Il y aurait alors de la cruauté sans que personne ne soit cruel. " (États d'âme..., 13, 89)

Jacques me dira plus tard, tout récemment, il y a quelques semaines, alors qu'il souffrait du mal dont il était atteint, que ces lignes avaient sans doute été écrites dans l'anticipation d'un savoir encore inconscient.

À la suite des États Généraux, la fête de l'esprit nous avait encore réservé de grands moments. À Montpellier en 2001, pour un colloque sur « La souveraineté » à la suite duquel le projet de Mélanges pour son $75^{\mathrm{e}}$ anniversaire fut mis en œuvre. Nous disposons d'ores et déjà des contributions qui seront publiées en juillet 2005 par les Éditions Galilée avec le concours, nous l'espérons, du Collège.

En juin 2001, nous avions participé ensemble à un Workshop à Rio qui fera également l'objet d'une publication, de même que la Controverse 
tenue en février 2003 à la Maison des Cultures du Monde sur le thème "Pourquoi la guerre ? ", à la veille de l'invasion de l'Irak, réunissant à la même table Derrida, Jean Baudrillard et Alain Gresh.

Une dernière grande fête, à laquelle j'ai eu le bonheur de participer avec plusieurs parmi nous, fut la belle réception d'un doctorat Honoris causa de l'Université de Coimbra, la plus ancienne université d'Europe, avec tout l'apparat, en cette circonstance, de sa vieille tradition. Ce fut l'occasion d'un colloque et de la réception par Jacques des clés de la ville qui devenait une Ville-refuge.

Jacques et moi, nous nous sommes toujours vouvoyés. Je le dis avant de livrer, dans l'indécence, un dernier mot, lorsque nous nous sommes vus pour la dernière fois à l'Institut Curie. C'était en présence de Marguerite et de Pierre. On l'avait soulagé de sa douleur. Il ne souffrait plus et la conversation se déroulait presque comme à l'accoutumé. Il fut question du Cahier de L'Herne qui venait de paraître et, bien sûr, d'analyse. Au moment de nous séparer, il me dit, sur un ton à la fois grave et enjoué : « Si je fais une analyse, René, ce sera avec vous. "Nous nous sommes quittés sur un long sourire. J'aime ce dernier mot pour sa promesse du futur (ce sera, quoi qu'il en soit) et son conditionnel qui n'exclut nullement l'inconditionnel (comme il aimait dire inconditionnellement « la déconstruction, s'il y en a » et « la psychanalyse, si une telle chose existe »).

" Le monde s'en va, le monde disparaît, Die Welt ist fort, Ich muss dich tragen, il me faut te porter, là où il n'y aurait plus de monde, pas encore le monde, là où le monde s'éloignerait, perdu au loin, encore à venir $[\ldots]$ " (Voyous, 213).

(Ce texte fut prononcé lors de l' "Hommage à Jacques Derrida » tenu au Collège international de philosophie, le 21 octobre 2004.)

\footnotetext{
${ }^{1}$ Paru d'abord dans la revue Contretemps, $\mathrm{n}^{\text {os }} 2 / 3$, Galilée, 1997, puis dans Voiles, avec Hélène Cixous.

${ }^{2}$ Dans Oxford Literary Review, vol. 12, n $1 / 2,1990$.
} 